\title{
THE STRUCTURE, PROTON AFFINITY, ELECTROSTATIC PROPERTIES AND ITS RELATION TO BIOLOGICAL ACTIVITY OF COCAINE AND ITS DERIVATIVES.
}

\author{
S. Roszak and A. Rzepiela
}

\author{
Institute of Physical and Theoretical Chemistry \\ Wroclaw University of Technology \\ Wyb. Wyspiańskiego 27 \\ 50-370 Wrocław, Poland
}

\begin{abstract}
.
Theoretical studies based on Hartree-Fock (HF) and Density Functional Theory (with the hybrid functional B 3 LYP) methods using a 6-31G basis set were used for (-)cocaine derivatives. Mulliken charges and proton affinities of selected atoms have been computed at the DFT level of theory for structures of molecules optimized at the HF level. The unequivocal relations have been found between proton affinities and available experimental data. The existence of at least two binding sites between active cocaine derivatives and the dopamine transporter has been confirmed.
\end{abstract}

I. Introduction.

Cocaine has several sites of action in the central nervous system. It has been shown [1] to block the reuptake of norepinephrine (NE), serotonin (5-HT), and dopamine (DA) as well as to exert effects on the cholinergic muscarinic, and a receptors. Any one or a combination of these could mediate effects related to the abuse of cocaine. At present, however it is mainly the dopaminergic pathway that has been implicated in the reinforcing properties of cocaine [2], In fact, the predominant theoretical base for the reinforcing properties of cocaine is the so called "dopamine hypothesis". This hypothesis assumes that cocaine binds to the dopamine transporter site in a way which inhibits dopamine transport. Thus, dopamine interaction (binding) with the dopamine receptors alters the functional state of limbic areas leading to the observed behavioural effects of cocaine. Little is known about the molecular mechanism by which the binding of cocaine to the sodium-dependent dopamine transporter leads to inhibition of reuptake. Despite gaps in knowledge regarding mechanism, it is reasonable to speculate that the compound capable of competing with cocaine for its binding sites on the dopamine transporter could modulate dopamine reuptake. Important insights into the interactions of cocaine with the dopamine transporter are provided by an understanding of the structure-activity correlation of cocaine. 
Several structure-activity studies have been reported [3-12] which have concluded the requirements for the cocaine binding. This analysis leads to the following observations [1]:

1. The largest factor in the activity of cocaine-like compounds is the absolute configuration. This effect is not carried over into the cocaine isomers.

2. The second largest factor is the substituent at C-2 [Fig. 1], The stereochemistry at C-2 has also large effect on the activity.

3. Substitution at nitrogen have a fairly large effect, in particular when it changes the electron density at nitrogen.

4. Significant and important effects on activity are obtained by substitution at C-3.

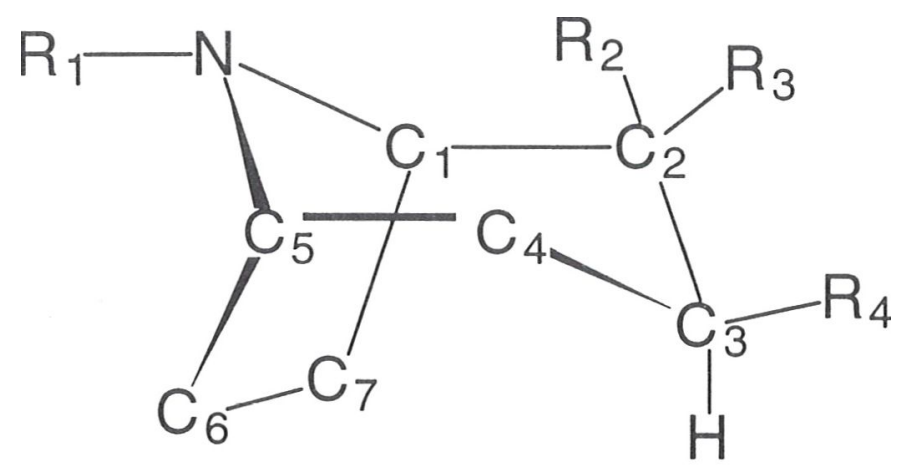

Fig. 1. The schematic representation of the (-) isomer of cocaine derivatives.

The structure-activity relation (SAR) data combined with some preliminary molecular modelling studies [6] allow speculation on a preliminary pharmacophore model for the cocaine receptor. All high-affinity cocaine ligands require the presence of a basic amino group which is involved in an electrostatic or hydrogen bonding interaction with the receptor protein. The SAR data concerning substituents at the C2-position of cocaine suggest the presence of at least one and probably two additional bond acceptor sites localized in the vicinity of the two oxygen's of the $2 \beta$ carbomethoxy group of cocaine. An aromatic ring connected directly or indirectly to the $3 \beta$ position of the tropane ring is required for good affinity of the receptor. The optimum location and properties of this binding side have not been defined. Present data indicate that hydrophobicity, charge and size are all important. From a steric standpoint the receptor can accommodate only small increases in size at the nitrogen position or on the aromatic ring at C-3. In contrast, large groups can replace the methyl of the carbomethoksy group at C-2 with very little loss in affinity for the receptor. The importance of structural sites and its properties indicated by 
experimental works of cocaine derivatives directs the further investigations toward theoretical studies.

Quantum chemistry enables the systematic study on the same level of sophistication for any property associated with the particular site of molecule.

The preliminary studies of the electronic charge distribution and proton affinities of cocaine derivatives are presented in this study.

II. Details of the Theoretical Treatment.

The structure of cocaine and its derivatives has been optimized at the Hartree-Fock level of theory. The proton position in protonated structures have also been optimized at the same level. The standard 6-31G basis set [13] has been utilized. The optimization of cocaine has been started using coordinates available from crystallographic studies [14]. The cocaine skeleton has been used to generate structures of 15 derivatives (Table 1) studied experimentally [15]. Theoretical and experimental bond distances and angles for (-)cocaine given in Table 2 agree well giving a confidence in the overall structure optimizations performed in this study.

Table 1. The analogues of cocaine, (see Figure 1.)

\begin{tabular}{|l|c|c|c|c|}
\hline Compound & $\mathrm{R}_{1}$ & $\mathrm{R}_{2}$ & $\mathrm{R}_{3}$ & $\mathrm{R}_{4}$ \\
\hline \hline Cocaine & $\mathrm{CH}_{3}$ & $\mathrm{COOCH}_{3}$ & $\mathrm{H}$ & $\mathrm{OCOC}_{6} \mathrm{H}_{5}$ \\
\hline Norcocaine & $\mathrm{H}$ & $\mathrm{COOCH}_{3}$ & $\mathrm{H}$ & $\mathrm{OCOC}_{6} \mathrm{H}_{5}$ \\
\hline Tropacocaine & $\mathrm{CH}_{3}$ & $\mathrm{H}$ & $\mathrm{H}$ & $\mathrm{OCOC}_{6} \mathrm{H}_{5}$ \\
\hline Pseudococaine & $\mathrm{CH}_{3}$ & $\mathrm{H}$ & $\mathrm{COOCH}_{3}$ & $\mathrm{OCOC}_{6} \mathrm{H}_{5}$ \\
\hline Benzoylecgonine & $\mathrm{CH}_{3}$ & $\mathrm{COOH}$ & $\mathrm{H}$ & $\mathrm{OCOC}_{6} \mathrm{H}_{5}$ \\
\hline $\begin{array}{l}\text { Meta-toluylecgonine } \\
\text { methyl ester }\end{array}$ & $\mathrm{CH}_{3}$ & $\mathrm{COOCH}_{3}$ & $\mathrm{H}$ & $\mathrm{OCOC}_{6} \mathrm{H}_{4} \mathrm{CH}_{3}$ \\
\hline $\begin{array}{l}\text { P-aminobenzoylecgonine } \\
\text { methyl ester }\end{array}$ & $\mathrm{CH}_{3}$ & $\mathrm{COOCH}_{3}$ & $\mathrm{H}$ & $\mathrm{C}_{6} \mathrm{H}_{4} \mathrm{NH}_{2}$ \\
\hline WIN 35,065 & $\mathrm{CH}_{3}$ & $\mathrm{COOCH}_{3}$ & $\mathrm{H}$ & $\mathrm{C}_{6} \mathrm{H}_{5}$ \\
\hline WIN 35,981 & $\mathrm{H}$ & $\mathrm{COOCH}$ & $\mathrm{H}$ & $\mathrm{C}_{6} \mathrm{H}_{5}$ \\
\hline WIN 35,428 & $\mathrm{CH}_{3}$ & $\mathrm{COOCH}$ & $\mathrm{H}$ & $\mathrm{C}_{6} \mathrm{H}_{4} \mathrm{~F}$ \\
\hline Ecgonine methyl ester & $\mathrm{CH}_{3}$ & $\mathrm{COOCH}$ & $\mathrm{H}$ & $\mathrm{OH}_{3}$ \\
\hline P-aminobenzoylecgonine & $\mathrm{CH}_{3}$ & $\mathrm{COOH}$ & $\mathrm{H}$ & $\mathrm{C}_{6} \mathrm{H}_{4} \mathrm{NH}_{2}$ \\
\hline $\begin{array}{l}\text { Pseudoecgonine } \\
\text { methyl ester }\end{array}$ & $\mathrm{CH}_{3}$ & $\mathrm{H}$ & $\mathrm{COOCH}_{3}$ & $\mathrm{OH}^{-}$ \\
\hline WIN 35,140 & $\mathrm{CH}_{3}$ & $\mathrm{H}$ & $\mathrm{COOCH}$ & $\mathrm{C}_{6} \mathrm{H}_{5}$ \\
\hline Ecgonine & $\mathrm{CH}_{3}$ & $\mathrm{COOH}$ & $\mathrm{H}$ & $\mathrm{OH}_{3}$ \\
\hline Benzoylnorecgonine & $\mathrm{H}$ & $\mathrm{COOH}$ & $\mathrm{H}$ & $\mathrm{OCOC}_{6} \mathrm{H}_{5}$ \\
\hline
\end{tabular}


The computations of molecular properties: atomic Mulliken charges and proton affinities have been performed at the density functional theory (DFT) level. The DFT approach has used the hybrid Becke's three parameter functional (B3LYP) [16] were the non-local correlation is provided by the Lee-Yang-Parr expression [17].

The DFT method, including large part of correlation effects, is superior to the HF approach and has been found as an efficient tool to study molecular properties [18].All the computations were made using the Gaussian 94 code [19].

Table 2. The HF/6-31G optimized bond distances (in angstrom) and bond angles (in degree) for the skeleton of (-)cocaine. Experimental results correspond to X-ray data [14].

\begin{tabular}{|c|c|c|c|c|c|}
\hline Bond Lengths & Theoretical & Experimental & Bond Angles & Theoretical & Experimental \\
\hline $\mathrm{C}_{1}-\mathrm{C}_{2}$ & 1.5495 & 1.5325 & $\mathrm{C}_{1}-\mathrm{C}_{2}-\mathrm{C}_{3}$ & 108.80 & 107.24 \\
\hline $\mathrm{C}_{2}-\mathrm{C}_{3}$ & 1.5280 & 1.5186 & $\mathrm{C}_{2}-\mathrm{C}_{3}-\mathrm{C}_{4}$ & 112.77 & 112.24 \\
\hline $\mathrm{C}_{3}-\mathrm{C}_{4}$ & 1.5278 & 1.5118 & $\mathrm{C}_{3}-\mathrm{C}_{4}-\mathrm{C}_{5}$ & 109.52 & 110.24 \\
\hline $\mathrm{C}_{4}-\mathrm{C}_{5}$ & 1.5363 & 1.5315 & $\mathrm{C}_{4}-\mathrm{C}_{5}-\mathrm{C}_{6}$ & 112.19 & 111.25 \\
\hline $\mathrm{C}_{5}-\mathrm{C}_{6}$ & 1.5557 & 1.5278 & $\mathrm{C}_{5}-\mathrm{C}_{6}-\mathrm{C}_{7}$ & 103.84 & 103.84 \\
\hline $\mathrm{C}_{6}-\mathrm{C}_{7}$ & 1.5534 & 1.5359 & $\mathrm{C}_{6}-\mathrm{C}_{7}-\mathrm{C}_{1}$ & 103.99 & 103.55 \\
\hline $\mathrm{C}_{7}-\mathrm{C}_{1}$ & 1.5543 & 1.5227 & $\mathrm{C}_{7}-\mathrm{C}_{1}-\mathrm{C}_{2}$ & 112.49 & 113.94 \\
\hline $\mathrm{C}_{1}-\mathrm{N}_{18}$ & 1.4676 & 1.4607 & $\mathrm{C}_{5}-\mathrm{N}_{18}-\mathrm{C}_{1}$ & 102.98 & 100.94 \\
\hline $\mathrm{C}_{5}-\mathrm{N}_{18}$ & 1.4737 & 1.4676 & $\mathrm{C}_{5}-\mathrm{N}_{18}-\mathrm{C}_{17}$ & 116.67 & 112.74 \\
\hline $\mathrm{N}_{18}-\mathrm{C}_{17}$ & 1.4570 & 1.4687 & $\mathrm{C}_{1}-\mathrm{N}_{18}-\mathrm{C}_{17}$ & 116.63 & 113.24 \\
\hline $\mathrm{C}_{2}-\mathrm{C}_{15}$ & 1.5075 & 1.5096 & $\mathrm{C}_{1}-\mathrm{C}_{2}-\mathrm{C}_{15}$ & 108.44 & 108.24 \\
\hline $\mathrm{C}_{15}-\mathrm{O}_{21}$ & 1.2104 & 1.1885 & $\mathrm{C}_{3}-\mathrm{C}_{2}-\mathrm{C}_{15}$ & 114.18 & 114.64 \\
\hline $\mathrm{C}_{15}-\mathrm{O}_{22}$ & 1.3528 & 1.3345 & $\mathrm{C}_{2}-\mathrm{C}_{15}-\mathrm{O}_{21}$ & 128.50 & 128.84 \\
\hline $\mathrm{O}_{22}-\mathrm{C}_{16}$ & 1.4441 & 1.4429 & $\mathrm{C}_{2}-\mathrm{C}_{15}-\mathrm{O}_{22}$ & 110.08 & 109.13 \\
\hline $\mathrm{C}_{3}-\mathrm{O}_{19}$ & 1.4477 & 1.4514 & $\mathrm{O}_{21}-\mathrm{C}_{15}-\mathrm{O}_{22}$ & 121.42 & 123.24 \\
\hline $\mathrm{O}_{19}-\mathrm{C}_{8}$ & 1.3440 & 1.3305 & $\mathrm{C}_{15}-\mathrm{O}_{22}-\mathrm{C}_{16}$ & 119.51 & 116.64 \\
\hline $\mathrm{C}_{8}-\mathrm{O}_{20}$ & 1.2194 & 1.1936 & $\mathrm{C}_{4}-\mathrm{C}_{3}-\mathrm{O}_{19}$ & 111.47 & 111.24 \\
\hline $\mathrm{C}_{8}-\mathrm{C}_{9}$ & 1.4749 & 1.4996 & $\mathrm{C}_{2}-\mathrm{C}_{3}-\mathrm{O}_{19}$ & 107.87 & 109.63 \\
\hline $\mathrm{C}_{9}-\mathrm{C}_{10}$ & 1.3917 & 1.3537 & $\mathrm{C}_{3}-\mathrm{O}_{19}-\mathrm{C}_{8}$ & 121.59 & \begin{tabular}{|l|}
117.63 \\
\end{tabular} \\
\hline $\mathrm{C}_{10}-\mathrm{C}_{11}$ & 1.3863 & 1.3837 & $\mathrm{O}_{19}-\mathrm{C}_{8}-\mathrm{O}_{20}$ & 122.15 & 123.84 \\
\hline $\mathrm{C}_{11}-\mathrm{C}_{12}$ & 1.3882 & 1.3569 & $\mathrm{O}_{19}-\mathrm{C}_{8}-\mathrm{C}_{9}$ & 113.08 & 111.44 \\
\hline $\mathrm{C}_{12}-\mathrm{C}_{13}$ & 1.3892 & \begin{tabular}{|l|}
1.3411 \\
\end{tabular} & $\mathrm{O}_{20}-\mathrm{C}_{8}-\mathrm{C}_{9}$ & 124.77 & 124.84 \\
\hline $\mathrm{C}_{13}-\mathrm{C}_{14}$ & 1.3844 & 1.3879 & $\mathrm{C}_{8}-\mathrm{C}_{9}-\mathrm{C}_{10}$ & 121.15 & 121.64 \\
\hline $\mathrm{C}_{14}-\mathrm{C}_{9}$ & 1.3917 & 1.3807 & $\mathrm{C}_{8}-\mathrm{C}_{9}-\mathrm{C}_{14}$ & 118.59 & 118.54 \\
\hline
\end{tabular}


Table 3. Mulliken atomic charges for selected atoms, dipole moments (in Debye) and experimental IC Fo $_{5}$ alues of inhibition of binding of ${ }^{3} \mathrm{H}$-mazindol at dopamine transporter for (-)cocaine derivatives. Theoretical calculations have been performed within the DFT(B3LYP)/6-31G method for HF/6-31G optimized geometries.

\begin{tabular}{|l|c|c|c|c|c|c|c|c|c|c|c|c|}
\hline Compound & $\mathrm{IC}_{50}$ & $\mathrm{~N}$ & $\mathrm{C}_{1}$ & $\mathrm{C}_{2}$ & $\mathrm{C}_{3}$ & $\mathrm{C}_{4}$ & $\mathrm{C}_{5}$ & $\mathrm{C}_{6}$ & $\mathrm{C}_{7}$ & $\mathrm{C}(\mathrm{R})_{2}$ & $\mathrm{O}(\mathrm{R})_{2}$ & $\mathrm{Dipole}$ \\
\hline \hline$(-)$ WIN 35,428 & 0.17 & -0.421 & -0.014 & -0.138 & -0.195 & -0.216 & -0.017 & -0.263 & -0.260 & 0.557 & -0.429 & 4.382 \\
\hline$(-)$ WIN 35,065 & 0.26 & -0.420 & -0.014 & -0.137 & -0.195 & -0.216 & -0.017 & -0.262 & -0.260 & 0.556 & -0.427 & 2.454 \\
\hline (-)WIN 35,981 & 0.36 & -0.513 & -0.039 & -0.132 & -0.195 & -0.210 & -0.040 & -0.253 & -0.254 & 0.558 & -0.426 & 2.716 \\
\hline (-)Cocaine & 0.64 & -0.418 & -0.023 & -0.153 & 0.119 & -0.222 & -0.025 & -0.268 & -0.265 & 0.570 & -0.421 & 3.049 \\
\hline (-)Norcocaine & 1.21 & -0.511 & -0.046 & -0.148 & 0.118 & -0.216 & -0.047 & -0.262 & -0.259 & 0.572 & -0.420 & 3.124 \\
\hline $\begin{array}{l}\text { P-aminobenzoylecgonine } \\
\text { methyl ester }\end{array}$ & 3.04 & -0.631 & -0.023 & -0.217 & -0.201 & -0.245 & -0.029 & -0.320 & -0.317 & 0.856 & -0.591 & 1.662 \\
\hline P-aminobenzoylecgonine & 4.51 & -0.420 & -0.015 & -0.129 & -0.196 & -0.214 & -0.016 & -0.262 & -0.259 & 0.516 & -0.407 & 1.333 \\
\hline Tropacocaine & 15.0 & -0.411 & -0.028 & -0.204 & 0.096 & -0.213 & -0.027 & -0.265 & -0.264 & - & - & 1.848 \\
\hline $\begin{array}{l}\text { (-)Meta-toluylecgonine } \\
\text { methyl ester }\end{array}$ & 40.0 & -0.418 & -0.024 & -0.152 & 0.119 & -0.222 & -0.025 & -0.268 & -0.264 & 0.569 & -0.421 & 3.192 \\
\hline$-(-)$ Ecgonine methyl ester & 62.0 & -0.426 & -0.014 & -0.164 & 0.112 & -0.223 & -0.020 & -0.262 & -0.263 & 0.571 & -0.457 & 3.888 \\
\hline $\begin{array}{l}\text { (-)Pseudoecgonine } \\
\text { methyl ester }\end{array}$ & 87.0 & -0.417 & -0.034 & -0.118 & 0.095 & -0.220 & -0.024 & -0.262 & -0.270 & 0.525 & -0.430 & 3.116 \\
\hline $\begin{array}{l}\text { (-)Pseudococaine } \\
\text { (-)Benzoylecgonine }\end{array}$ & 97.0 & -0.413 & -0.033 & -0.117 & 0.106 & -0.216 & -0.030 & -0.264 & -0.274 & 0.519 & -0.425 & 3.383 \\
\hline WIN 35,140 & 342 & -0.513 & -0.046 & -0.143 & 0.117 & -0.217 & -0.046 & -0.262 & -0.259 & 0.532 & -0.398 & 2.638 \\
\hline (-)Benzoylnorecgonine & 385 & -0.417 & -0.027 & -0.105 & -0.203 & -0.205 & -0.021 & -0.259 & -0.269 & 0.513 & -0.433 & 1.816 \\
\hline (-)Ecgonine & 1000 & -0.420 & -0.023 & -0.148 & 0.118 & -0.222 & -0.024 & -0.268 & -0.264 & 0.529 & -0.398 & 2.538 \\
\hline
\end{tabular}

\footnotetext{
a) the increase of IC value indicates the reduction of potency in inhibiting the ${ }^{3} \mathrm{H}$-mazindol binding, ref. [15],
} 
III. Results and discussion.

The Muiliken population analysis gives the representation of electronic density distribution in the molecule. The calculated Muiliken charges are shown in Table 3. Its relation to the biological activity is rather weak. For the important position: the oxygen atom in the $\mathrm{CO}$ group attached to carbon $\mathrm{C} 2$ atomic charges have similar values for active cocaine derivatives. These charges are not unique however and can also be found in some inactive molecules.

Table 4. The proton affinities (in eV) for oxygen in the carboxyl group attached to $\mathrm{C} 2$ carbon and for $\mathrm{N}$ of cocaine ring and experimental $\mathrm{IC}_{50}$ values (see Table 3 for description) for (-)cocaine derivatives. Theoretical calculations have been performed within the DFT(B3LYP)/6-31G method for HF/6-31G optimized geometries.

\begin{tabular}{|l|c|c|c|}
\hline Compound & $\mathrm{IC}_{50}$ & $\mathrm{PA}(\mathrm{O})$ & $\mathrm{PA}(\mathrm{N})$ \\
\hline \hline$(-)$ WIN 35,428 & 0.17 & 8.54 & 10.48 \\
\hline$(-)$ WIN 35,065 & 0.26 & 8.69 & 10.60 \\
\hline (-)WIN 35,981 & 0.36 & 8.66 & 10.47 \\
\hline (-)Cocaine & 0.64 & 8.52 & 10.41 \\
\hline (-)Norcocaine & 1.21 & 8.52 & 10.27 \\
\hline P-aminobenzoylecgonine methyl ester & 3.04 & 8.77 & 10.74 \\
\hline P-aminobenzoylecgonine & 4.51 & 8.48 & 10.65 \\
\hline Tropacocaine & 15.0 & - & 10.51 \\
\hline (-)Meta-toluylecgonine methyl ester & 40.0 & 8.53 & 10.43 \\
\hline (-)Ecgonine methyl ester & 62.0 & 8.30 & 10.32 \\
\hline (-)Pseudoecgonine methyl ester & 87.0 & 8.42 & 5.41 \\
\hline (-)Pseudococaine & 97.0 & 8.37 & 10.38 \\
\hline$(-)$ Benzoylecgonine & 342 & 8.77 & 10.17 \\
\hline WIN 35,140 & 385 & 8.49 & 10.43 \\
\hline$(-)$ Benzoylnorecgonine & 392 & 8.21 & 10.32 \\
\hline (-)Ecgonine & 1000 & 7.98 & 10.21 \\
\hline
\end{tabular}

The proton affinities have been calculated as an energy difference between protonated and deprotonated forms of cocaine derivatives $\left(\mathrm{PA}=\mathrm{E}_{\mathrm{COCH}+}-\mathrm{E}_{\mathrm{COC}}\right)-\mathrm{Two}$ possible protonation sites indicated by experimental findings have been studied: with proton attached to oxygen of the $\mathrm{C}=0$ fragment of the $\mathrm{R}_{2}$ or $\mathrm{R}_{3}$ group and with the proton attached to nitrogen present in the cocaine ring. The unequivocal correlation 
has been found between theoretical proton affinities and the experimental measure of biological activity (Table 4).

Affinities are high for active derivatives. Both $\mathrm{O}$ and $\mathrm{N}$ sites are important. Theoretical results confirm earlier experimental findings which suggest the existence of at least two ionic or $\mathrm{H}$-bond interaction sites. The presented preliminary results indicate the usefulness of applied theoretical approaches.

The close agreement between calculated and available X-ray structure of cocaine give a confidence in the overall set of theoretical structures of molecules.

Proton affinities (being the measure of reactivity) calculated for atoms indicated as possible sites of interaction of cocaine with its receptor at the dopamine transporter correlates well with the experimental biological activity. The electron density distribution, described by Mulliken atomic charges, is not correlated with an activity of molecules in a straightforward manner.

\section{ACKNOWLEDGEMENTS.}

The authors thank Prof. W. A. Sokalski for reading of the manuscript and valuable comments. This work was supported in part by the State Committee for Scientific Research grant No. 6 P04A 060 09. We wish to thank the Poznań Supercomputing and Networking Center for providing computer time.

Bibliography:

1. F. I. Carroll, A. H. Levin, J. W. Boja and M. J. Kuhar, J. Med. Chem. 35, 969 (1992).

2. M. J. Kuchar, M. C. Ritz and J. W. Boja, Trends in Neuroscience 14, 299 (1991).

3. F. I. Carroll, A. H. Levin, P. Abraham, K. Parham, J. W. Boja and M. J. Kuhar, J. Med. Chem. 34, 883 (1991).

4. M. E. A. Reith, B. E. Meisler, H. Sershen and A. Lajtha, Biochem. Pharmacol. 35, 1123 (1986).

5. M. C. Ritz, E. J. Cone and M. J. Cone, Life Sci. 46, 635 (1990).

6. F. I. Carroll, Y. Gao, M. A. Rahman, P. Abraham, K. Parham, A.H. Levin, J. W. Boja and M. J. Kuhar, J. Med. Chem. 34, 2719 (1991).

7. P. Abraham, J. B. Pitner, A. H. Levin, J. W. Boja, M. J. Kuhar and F. I. Carroll, J. Med. Chem. 35, 141 (1992).

8. A. H. Lewin, Y. Gao, P. Abracham, J. W. Boja, M. J. Kuhar and F. I. Carroll, J. Med. Chem. 35, 135 (1992). 
9. A. P. Kozikowski, M. Roberti, L. Xiang, J. S. Bergmann, P. M. Callahan, K. A. Cunningham and K. M. Johnson, J. Med. Chem. 35, 4764 (1994).

10. G. S. Schuelke, L. C. Terry, R. H. Powers, J. Rice and J. A. Madden, Pharm. Biochem. Behavior 53, 133 (1966).

11. H. M. Deutsch, Q. Shi, E. Gruszecka-Kowalik and M. M. Schweri, J. Med. Chem. 39, 1201 (1996).

12. A. E. Fleckenstein, T. A. Kopajtic, J. W. Boja, F. I. Carroll and M. J. Kuhar, Eur. J. Pharmacol. 311, 109 (1996).

13. R. Ditchfield, W. J. Hehre and J. A. Pople, J. Chem. Phys. 54, 724 (1971).

14. R. J. Hrynchuk, R. J. Barton and B. E. Robertson, Can. J. Chem. 61, 481 (1983).

15. M. C. Ritz, E. J. Cone and M. J. Kuchar, Life Sei. 46, 635 (1990).

16. A. D. Becke, J. Chem. Phys. 98, 5648 (1993).

17. C. Lee, W. Yang and R. G. Parr, Phys. Rev. B37, 785 (1988).

18. Materials of , $7^{\text {lh }}$ International Conference on the Applications of the Density Functional Theory in Chemistry and Physics", Technical University, Vienna, 1997.

19. Gaussian 94, Revision C.2, M. J. Frish, G. W. Trucks, H. B. Schlegel, P. M. W. Gill, B. G. Johnson, M. A. Robb, J. R. Cheeseman, T. Keith, G. A. Petersson, M. A. Robb, J. A. Montgomery, K. Raghavachari, M. A. Al-Laham, V.G.

Zakrzewski, J. V. Oritz, J. B. Foresman, J. Cioslowski, B. B. Stefanov, A. Nanayakkara, M. Challacombe, C. Y. Peng, P. Y. Ayala, W. Chen, M. W. Wong, J. L. Andres, E. S. Replogle, R. Gomperts, R. L. Martin, D. J. Fox, J. S. Binkley, D. J. Defrees, J. Baker, J. P. Stewart, M. Head-Gordon, C. Gonzalez, and J.A. Pople, Gaussian Inc., Pittsburgh, PA, 1995. 